\title{
Theoretical Characterization of the Resonance-Energy Transfer Between Organic Molecules
}

\author{
Demétrio A. S. Filho
}

\section{Introduction}

One of the most interesting processes that occur in organic materials is the resonance-energy transfer. This process can be useful to further enhance the efficiency of devices based on organic molecules. Here we will investigate theoretically the resonance-energy transfer (RET) process between a para-hexaphenyl (p6P) unit and a $\alpha$-sexithiophene (6T) unit.

\section{Methods}

Theoretical calculations using Density Functional Theory (DFT) and its Time-Dependent approach (TDDFT) were also carried out in order to obtain equilibrium energies, geometries and frequencies for both ground $\left(\mathrm{S}_{0}\right)$ and first excited $\left(\mathrm{S}_{1}\right)$ states of $\mathrm{p} 6 \mathrm{P}$ and $6 \mathrm{~T}$. These properties allow us to better understand the mechanisms behind the absorption and emission processes involved in the isolated molecules and to build a qualitative model to understand the energy transfer in the p6P/6T complex.

Geometries, frontier orbitals and total energies of ground state $\left(\mathrm{S}_{0}\right)$ and first excited state $\left(\mathrm{S}_{1}\right)$ of p6P and 6T were obtained using DFT. We make use of two different DFT functionals: i) the M06-2X functional, [1,2] which has proven to properly describe the spectroscopic properties of these type of systems[2,3] and ii) the CAM-B3YP functional, that has a good track record in the description of the electronic processes in organic molecules.[4,5]
The 6-31G(d) basis set was used for all calculations presented here. This basis set was chosen due to the relative large size of the systems under investigation in addition to the successful reproduction of the absorption and emission data of short oligomers.[5,6] All simulations were carried out using the Gaussian'09 program suite.[7]

\section{Results and Discussion}

Figure 1 shows the ground state (S0) HOMO (highest occupied molecular orbital) and LUMO (lowest unoccupied molecular orbitals) wavefuction for both p6P and 6T.

In solid state, both p6P and $6 \mathrm{~T}$ are observed to be linear and planar molecules, in both ground and excited states. Thus, in order to better compare the experimental results with our calculations, we also optimized p6P and 6T subjected to the constraint of being planar, and evaluated the impact of this constraint in the $\mathrm{S} 0 \rightarrow \mathrm{S} 1$ transition energy, which is also included in Figure 1b.

The impact of planarization is larger on p6P than on $6 \mathrm{~T}$, as expected from the larger deviation from planarity presented by the former oligomer. A comparison between the HOMO-LUMO gap of the two planar structures shows that p6P has still a larger gap compared to 6T, but the difference between the two has reduced from $1.39 \mathrm{eV}$, in the twisted form, to $0.46 \mathrm{eV}$ in the planar (solid-state like) form.

The results for M06-2X shows a similar trend, although the mismatches between the gap of p6P and $6 \mathrm{~T}$ were computed to be $1.37 \mathrm{eV}$ and $0.837 \mathrm{eV}$, respectively. 
This small difference (on the order of tenths of $\mathrm{eV}$ ) in the HOMO-LUMO gap and the fact that p6P has a larger gap compared to $6 \mathrm{~T}$ already suggests that $\mathrm{p} 6 \mathrm{P}$ can absorb a higher energy photon and transfer this energy to $6 \mathrm{~T}$, as it was observed for other thiophene-based systems.

To further characterize this energy transfer, a simulation of the vibronic resolved absorption spectrum of $6 \mathrm{~T}$ together with the counterpart emission spectrum of p6P must be carried out.

a)

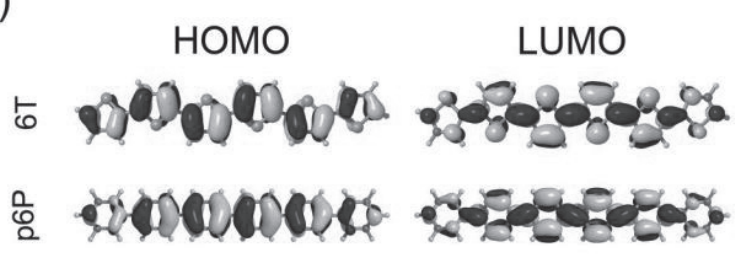

b)

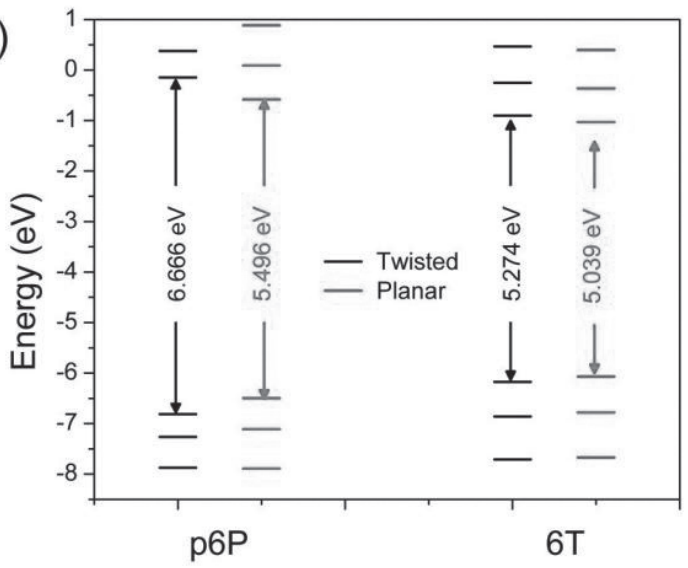

Figure 1. a) 6T (top) and p6P (bottom) ground state HOMO (left) and LUMO (right) wavefunctions. (b) CAM-B3LYP ground state (S0) frontier orbital energies and HOMO-LUMO gap for p6P (left) and 6T (right). Both fully optimized (twisted) and optimized under the planarity constraint (planar) are presented.

\section{Conclusions}

Theoretical calculations in the framework of Density Functional Theory were used to characterize the potential energy surfaces of the ground (S0) and first excited (S1) states of p6P and 6T. By a comparison between the energy gap related to the emission of $\mathrm{p} 6 \mathrm{P}$ and the absorption of $6 \mathrm{~T}$ from their lowest vibronic state, we determined a mismatch of $0.2 \mathrm{eV}$ of the order of the vibrational relaxation energy of the molecules, resulting in a high probability for resonance-energy transfer between the two molecules.

\section{Acknowledgments}

D. A. S. Filho gratefully acknowledges the financial support from the Brazilian Research Councils: CAPES, CNPq (grant 306968/2013-4) and FAP-DF (Fundação de Apoio à Pesquisa do Distrito Federal).

\section{References}

1. Zhao, Y.; Truhlar, D. G. The M06 Suite of Density Functionals for Main Group Thermochemistry, Thermochemical Kinetics, Noncovalent Interactions, Excited States, and Transition Elements: Two New Functionals and Systematic Testing of Four M06-Class Functionals and 12 Other Function. Theor. Chem. Acc. 2008, 120, 215-241.

2. Zhao, Y.; Truhlar, D. G. The M06 Suite of Density Functionals for Main Group Thermochemistry, Thermochemical Kinetics, Noncovalent Interactions, Excited States, and Transition Elements: Two New Functionals and Systematic Testing of Four M06-Class Functionals and 12 Other Function. Theor. Chem. Acc. 2008, 119, 525.

3. Lumpi, D.; Horkel, E.; Plasser, F.; Lischka, H.; Fröhlich, J. Synthesis, Spectroscopy, and Computational Analysis of Photoluminescent Bis(aminophenyl)-Substituted Thiophene Derivatives. ChemPhysChem 2013, 14, 1016-1024.

4. Charaf-Eddin, A.; Planchat, A.; Mennucci, B.; Adamo, C.; Jacquemin, D. Choosing a Functional for Computing Absorption and Fluorescence Band Shapes with TD-DFT. J. Chem. Theory Comput. 2013, 9, 2749-2760.

5. Jacquemin, D.; Planchat, A.; Adamo, C.; Mennucci, B. TD-DFT Assessment of Functionals for Optical 0-0 Transitions in Solvated Dyes. J. Chem. Theory Comput. 2012, 8, 2359-2372.

6. Da Silva Filho, D. A.; Friedlein, R.; Coropceanu, V.; Ohrwall, G.; Osikowicz, W.; Suess, C.; Sorensen, S. L.; Svensson, S.; Salaneck, W. R.; Brédas, J.-L. Vibronic Coupling in the Ground and Excited States of the Naphthalene Cation. Chem. Commun. (Camb). 2004, $1702-1703$.

7. Frisch M. J.; Trucks, G. W.; Schlegel, H. B.; Scuseria, G. E.; Robb, M. A.; Cheeseman, J. R.; Scalmani, G.; Barone, V.; Mennucci, B.; Petersson, G. A.; et al. Gaussian 09, Revision A.02, 2009 S. W.Benson and O. Dobis, J. Phys. Chem. A,102 , 5175, (1998).

\section{Demétrio A. S. Filho}

Institute of Physics, University of Brasilia, 04455, DF, 70919-970, Brazil 